\title{
Preoperative Lymphocyte-Monocyte Ratio Is a Predictor of Suboptimal Cytoreduction in Stage III-IV Epithelial Ovarian Cancer
}

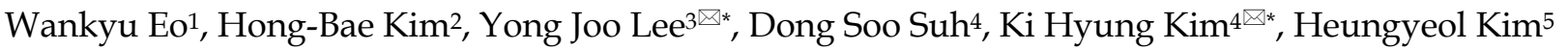 \\ 1. Department of Internal Medicine, College of Medicine, Kyung Hee University, Seoul, Korea; \\ 2. Department of Obstetrics and Gynecology, Kangnam Sacred Heart Hospital, Hallym University Medical Center, Hallym University College of Medicine, \\ Seoul, Korea; \\ 3. Department of Medicine, Pusan National University Graduate School; \\ 4. Department of Obstetrics and Gynecology, Pusan National University School of Medicine; Biomedical Research Institute, Pusan National University \\ Hospital, Busan, Korea; \\ 5. Department of Obstetrics and Gynecology, College of Medicine, Kosin University, Busan, Korea.
}

*These authors equally contributed to this work.

$\square$ Corresponding authors: Yong Joo Lee, M.D., Ph.D., Department of Medicine, Pusan National University Graduate School, Busan 50612, Korea; Phone: +82-51-510-8004; Fax: +82-51-510-8026; e-mail: yongjooleepnu@yahoo.com Or Ki Hyung Kim, M.D., Ph.D., Department of Obstetrics and Gynecology, Pusan National University School of Medicine; Biomedical Research Institute, Pusan National University Hospital, Busan 602-739, Korea; Phone: +82-51-990-6226; Fax: +82-51-990-3300; e-mail: kihyungkimpnu@gmail.com.

() Ivyspring International Publisher. Reproduction is permitted for personal, noncommercial use, provided that the article is in whole, unmodified, and properly cited. See http://ivyspring.com/terms for terms and conditions.

Received: 2016.04.03; Accepted: 2016.06.29; Published: 2016.08.11

\begin{abstract}
Objective: To determine whether the preoperative lymphocyte-monocyte ratio (LMR) is a predictor of suboptimal cytoreduction in advanced-stage epithelial ovarian cancer (EOC).

Methods: Preoperative clinico-pathologic and hematologic parameters were reviewed in a total of 154 patients with EOC submitted to primary cytoreductive surgery. Patients were categorized into two different groups according to the results of cytoreductive surgery: optimal and suboptimal cytoreduction. Continuous variables were categorized into two groups using the best cutoff points selected on the receiver operating characteristic (ROC) curve for suboptimal cytoreduction.

Results: Based on data collected from the 154 patients, 133 (86.4\%) and 21 (13.6\%) patients presented with stage III and IV disease, respectively. One hundred seventeen (76.0\%) patients had serous adenocarcinoma, and $92(59.7 \%)$ had histologic tumor grade 3 . The optimal and suboptimal cytoreduction groups included $96(62.3 \%)$ and 58 patients (37.7\%), respectively. The best LMR cutoff point for suboptimal cytoreduction was 3.75 . On multivariate logistic regression analysis, age, cancer antigen 125, white blood cell count, and LMR were found to be the strongest predictors for suboptimal cytoreduction $(P=0.0037,0.0249,0.0062$, and 0.0015 , respectively).

Conclusion: Preoperative LMR is an independent predictor of suboptimal cytoreduction. It provides additional prognostic information beyond the biological parameters of the tumor.
\end{abstract}

Key words: Lymphocytes, Monocytes, Cytoreduction Surgical Procedures, Ovarian neoplasms.

\section{Introduction}

Ovarian cancer remains one of the leading causes of death from gynecologic cancer worldwide [1]. One of the reasons for this dismal prognosis is that, on most occasions, ovarian cancer is diagnosed at an advanced stage [2]. To improve survival of advanced ovarian cancer patients, cytoreductive surgery to achieve a largest residual tumor mass of 1 $\mathrm{cm}$ or less in maximum diameter (e.g., optimal cytoreduction) followed by postoperative chemotherapy is recommended [3,4]. However, if 
patients fail to achieve optimal cytoreduction, they may experience major morbidity without survival benefit [5]. In addition, interval debulking surgery has not been considered to be useful for patients with a largest residual tumor more than $1 \mathrm{~cm}$ in maximum diameter after primary surgical cytoreduction [6]. To overcome situations in which it is difficult to achieve optimal cytoreduction, interval cytoreduction following neoadjuvant chemotherapy (NAC) has been suggested as an alternative treatment to primary cytoreductive surgery plus postoperative chemotherapy $[2,5]$. Therefore, the precise pretreatment discrimination of patients' not amenable to optimal cytoreduction may be one of the principal determinants of prognosis in ovarian cancer [7].

Several researchers have endeavored to identify the specific predictors of cytoreductive outcomes. With respect to age, optimal cytoreduction can be easily accomplished in young patients $[8,9]$. There is some evidence that inherent differences in tumor biology are associated with the resectability of tumors. For example, cytoreduction has been easily achieved in patients with low-grade tumors and in those without ascites [8]. In addition, molecular methods such as microarrays [10] and p53 gene expression determination [11] have been used to predict which patients can be optimally debulked. Furthermore, numerous studies have revealed that preoperative cancer antigen 125 (CA125) level is associated with the results of cytoreductive surgery [9, 12-15]. Specific laparoscopic [16], computed tomography (CT) $[9,17,18]$, and positron emission tomography/computed tomography (PET/CT) findings [19-21] have been suggested as means by which the prediction of cytoreductive surgery outcomes can be improved. Although several studies have suggested potential tools for predicting outcomes of cytoreductive surgery, controversial results have also been reported, and thus their use as valuable predictors in the clinic is limited. Finally, the expertise of the surgeon cannot be ignored [22]. Currently no certain set of predictors perform well enough for clinical purposes.

Recently, the LMR, the ratio of absolute lymphocyte count [ALC] to the absolute monocyte count [AMC]), has been shown to be associated with survival in patients with malignant lymphomas [23] and different types of solid tumors, including ovarian cancer [24]. In a previous study, we revealed the value of LMR in predicting overall survival (OS) of stage I to IV epithelial ovarian cancer (EOC) [24]. The purpose of this study is to evaluate whether LMR could serve as a predictor of suboptimal surgical cytoreduction in patients with stage IIII-IV EOC.

\section{Methods}

We retrospectively assessed 154 patients with stage III-IV EOC who underwent up-front cytoreductive surgery by highly trained surgeons at university hospitals from July 2003 to January 2015. Patients with synchronous cancers or prior cancers diagnosed within 5 years of study were excluded from the present study. Those who had been treated with NAC or any type of radiotherapy were also excluded. In addition, patients who had concurrent autoimmune disorders including rheumatoid arthritis, Sjogren syndrome, and Hashimoto's thyroiditis, or had evidence of active infection were excluded. In our study, 181 patients were initially included in the study. From them, we excluded those with histology not compatible with EOC (15), those without available tissue for review (3), those who did not undergo surgical resection (1), those with stage I-II EOC (4), and those with no available preoperative complete blood count (CBC) with differential count (4). Ultimately, 154 patients were included in this work. This study is not a subgroup analysis of our previous study on ovarian cancer [24]. Retrospective review, collection, and analysis of medical records were approved by the independent ethics committee before it was initiated. This study was performed in accordance with the ethical principles in the Declaration of Helsinki.

Clinico-pathologic variables including age, histological classification, histologic tumor grade, International Federation of Gynecology and Obstetrics (FIGO) stage, maximal diameter of residual tumor, ascites, serum albumin concentration, CA125 levels, and hematologic parameters (peripheral blood white blood cells [WBC], hemoglobin, platelet counts, absolute neutrophil count [ANC], AMC, and ALC) were obtained retrospectively from medical records of patients. In addition, the ratio of the ANC to the ALC (NLR) and the ratio of the platelet count to the ALC (PLR) were calculated. Only those laboratory measurements that have been performed prior to resection as part of the routine evaluation of patients were collected for review. If multiple preoperative laboratory test results were available, the one that was performed on the nearest date before the surgery was selected for review. When collecting laboratory results, we also considered the reference range and quality control in each university hospital. Histologic type classification was reviewed for consistency.

In this work, we defined optimal cytoreduction as a largest residual tumor mass of $1 \mathrm{~cm}$ or less in maximum diameter, in accordance with the definition of the Gynecologic Oncology Group (GOG) [25], and suboptimal cytoreduction as surgical exploration with residual tumor more than $1 \mathrm{~cm}$ in maximum 
diameter. All visible tumor tissue was removed if technically feasible to achieve optimal surgical cytoreduction [10]. Cytoreductive surgery was performed by experienced surgeons. These are faculty members in the departments of Obstetrics and Gynecology at several university hospitals [8, 26]. After an incision was made, ascites was collected for cytological evaluation. The surgical procedures included total hysterectomy with bilateral salpingo-oophorectomy, radical peritonectomy, diaphragmatic stripping, omentectomy, bowel resection, appendectomy, splenectomy, bilateral pelvic and paraaortic lymph node dissection, and resection of any other organ to which the tumor had spread.

Table 1. Characteristics of the 154 patients with epithelial ovarian cancer.

\begin{tabular}{ll}
\hline Variable & median (range) \\
\hline Age (years) & $56(26-84)$ \\
Histology, $n$ (\%) & $117(76.0)$ \\
$\quad$ Serous & $15(9.7)$ \\
$\quad$ Endometrioid & $10(6.5)$ \\
$\quad$ Mucinous & $6(3.9)$ \\
$\quad$ Clear cell & $2(1.3)$ \\
$\quad$ Mixed epithelial & $4(2.6)$ \\
$\quad$ Other & \\
Histologic grade, $n(\%)$ & $14(9.1)$ \\
$\quad$ G1 & $45(29.2)$ \\
G2 & $92(59.7)$ \\
G3 & $3(1.9)$ \\
$\quad$ Not available & \\
FIGO Stage, $n(\%)$ & $133(86.4)$ \\
III & $21(13.6)$ \\
IV & \\
Optimal cytoreduction, $n(\%)$ & $58(37.7)$ \\
$\quad$ No & $96(62.3)$ \\
$\quad$ Yes & \\
Ascites, $n(\%)$ & $34(22.1)$ \\
$\quad$ No & $117(76.0)$ \\
Yes & $3(1.9)$ \\
Not available & $597.5(7.6-50000.0)$ \\
CA125 (unit/mL) & $3.9(1.8-5.0)$ \\
Albumin $(\mathrm{g} / \mathrm{dL})$ & $7025(1530-18000)$ \\
WBC (per $\mu \mathrm{L})$ & $5053.7(1153.6-15480.0)$ \\
ANC (per $\mu \mathrm{L})$ & $431.7(71.2-2003.4)$ \\
AMC (per $\mu \mathrm{L})$ & $79.9(0-1431.8)$ \\
AEC (per $\mu \mathrm{L})$ & $1334.3(212.7-5215.9)$ \\
ALC (per $\mu \mathrm{L})$ & $12.2(5.2-15.6)$ \\
Hemoglobin $(\mathrm{g} / \mathrm{dL})$ & $342(104-1144)$ \\
Platelet $(x$ 10 $/ \mu \mathrm{L})$ & $3.9(0.7-24.9)$ \\
NLR & $245.5(33.9-1823.5)$ \\
PLR & $3.0(1.0-12.0)$ \\
LMR & \\
\hline
\end{tabular}

FIGO, The International Federation of Gynecology and Obstetrics; CA125, cancer antigen 125; WBC, white blood cell; ANC, absolute neutrophil count; AMC, absolute monocyte count; AEC, absolute eosinophil count; ALC, absolute lymphocyte count; NLR, neutrophil-lymphocyte ratio; PLR, platelet-lymphocyte ratio; LMR, lymphocyte- monocyte ratio.
Patients were dichotomized into the following two groups in accordance with the results of cytoreduction: optimal and suboptimal cytoreduction. Differences in variables between the two groups were assessed. The best cutoff point to obtain suboptimal cytoreduction was determined using receiver operating characteristic (ROC) curve analysis. Independent $t$-tests were used to compare the means of 2 independent groups, whereas chi-squared tests were used to determine the relationship between 2 categorical variables. Both univariate and multivariate logistic regression of variables were used to evaluate which parameters were predictors of suboptimal cytoreduction. In multivariate logistic regression, we included only those variables with $P$-values less than 0.05 , and performed backward stepwise analysis. All presented $P$-values are two-sided. If the $P$-value was less than 0.05 , statistical significance was assigned. Data analysis was performed with SPSS version 18.0 for windows (SPSS Inc., Chicago, IL, USA).

\section{Results}

The characteristics of the patients used in our cohort are summarized in Table 1 . One hundred seventeen $(76.0 \%)$ patients had serous adenocarcinoma, and 92 (59.7\%) had histologic tumor grade 3 in our cohort. In total, $133(86.4 \%)$ patients had stage III disease and $21(13.6 \%)$ had stage IV disease. Ascites was observed in 117 (76.0\%) patients. The median serum CA125 concentration was 597.5 units/mL. The median counts of WBC, ANC, AMC, and ALC were 7025, 5053.7, 431.7, and $1334.3 / \mu \mathrm{L}$, respectively. The median concentration of hemoglobin was $12.2 \mathrm{~g} / \mathrm{dL}$. The median platelet count was $342000 / \mu \mathrm{L}$. Finally, the median values of NLR, PLR, and LMR were 3.9, 245.5, and 3.0, respectively.

Regarding the stratification of patients with EOC based on the results of cytoreduction, the optimal and suboptimal cytoreduction groups included 96 (62.3\%) and $58(37.7 \%)$ patients, respectively. We evaluated differences in the baseline characteristics according to the two cytoreduction groups. Significant differences in means between two groups were observed for the following variables: ALC $(P=0.009), \operatorname{PLR}(P=0.040)$, and LMR $(P=0.036)$. In addition, a significant difference in FIGO stage was found between the two groups using the chi-squared test $(P=0.026)$ (Table 2$)$.

Using data from all eligible patients, the best LMR cutoff point to obtain suboptimal cytoreduction was found to be 3.75 (sensitivity: $79.31 \%$, specificity: 48.96\%, AUC: $0.593, P=0.0438$ ) based on ROC curve analysis. Univariate analysis of logistic regression for suboptimal debulking identified the following significant variables: age $(P=0.0056), \quad$ FIGO stage $(P=0.0172)$, CA125 concentration $(P=0.0149)$, WBC 
$(P=0.0289)$, ALC $(P=0.0122)$, NLR $(P=0.0225), P L R$ $(P=0.0015)$, and LMR $(P=0.0006)$. Using multivariate logistic regression, age (hazard ratio $[\mathrm{HR}]=5.98,95 \%$ confidence interval $[\mathrm{CI}]=1.79-20.03, P=0.0037)$, CA125 $(\mathrm{HR}=2.55,95 \% \mathrm{CI}=1.13-5.77, P=0.0249)$,
WBC $(\mathrm{HR}=0.32,95 \% \mathrm{CI}=0.14-0.72, P=0.0062)$, and LMR (HR $=0.27,95 \% \mathrm{CI}=0.12-0.61, P=0.0015)$ were found to be the strongest predictors for suboptimal cytoreduction (Table 3, Figure 1).

Table 2. Patient characteristics in accordance to the result of cytoreduction in the 154 patients with epithelial ovarian cancer

\begin{tabular}{|c|c|c|c|c|c|c|}
\hline \multirow[t]{2}{*}{ Variable } & & \multicolumn{2}{|c|}{ Optimal cytoreduction } & \multicolumn{2}{|c|}{ Suboptimal cytoreduction } & \multirow[t]{2}{*}{$P$-value } \\
\hline & & $n(\%)$ & Mean \pm SD & $n(\%)$ & Mean \pm SD & \\
\hline Age (years) & & 96 & $56.7 \pm 10.8$ & 58 & $58.6 \pm 13.6$ & 0.342 \\
\hline \multirow[t]{2}{*}{ Histology } & Serous & $72(75.0)$ & & 45 (77.6) & & 0.866 \\
\hline & Non-serous & $24(25.0)$ & & $13(22.4)$ & & \\
\hline \multirow{2}{*}{ Histologic grade } & G1 & $9(9.5)$ & & $5(8.9)$ & & 1.000 \\
\hline & G2/G3 & $86(90.5)$ & & $51(91.1)$ & & \\
\hline \multirow[t]{2}{*}{ FIGO stage } & III & $88(91.7)$ & & 45 (77.6) & & 0.026 \\
\hline & IV & $8(8.3)$ & & $13(22.4)$ & & \\
\hline \multirow[t]{2}{*}{ Ascites } & No & $24(25.3)$ & & $10(17.9)$ & & 0.395 \\
\hline & Yes & $71(74.7)$ & & $46(82.1)$ & & \\
\hline CA125 (unit/mL) & & 96 & $1086.7 \pm 1409.6$ & 58 & $2824.8 \pm 7250.9$ & 0.076 \\
\hline Albumin (g/dL) & & 96 & $3.8 \pm 0.7$ & 58 & $3.8 \pm 0.7$ & 0.619 \\
\hline WBC (per $\mu \mathrm{L})$ & & 96 & $7885.8 \pm 2877.3$ & 58 & $7359.1 \pm 2231.4$ & 0.206 \\
\hline ANC (per $\mu \mathrm{L}$ ) & & 96 & $5648.6 \pm 2689.6$ & 58 & $5435.3 \pm 2189.1$ & 0.611 \\
\hline $\mathrm{AMC}($ per $\mu \mathrm{L})$ & & 96 & $488.8 \pm 267.5$ & 58 & $464.5 \pm 209.5$ & 0.531 \\
\hline $\mathrm{AEC}($ per $\mu \mathrm{L})$ & & 96 & $113.9 \pm 107.9$ & 58 & $127.8 \pm 208.2$ & 0.639 \\
\hline ALC (per $\mu \mathrm{L})$ & & 96 & $1565.6 \pm 779.9$ & 58 & $1284.4 \pm 542.0$ & 0.009 \\
\hline $\mathrm{Hb}(\mathrm{g} / \mathrm{dL})$ & & 96 & $12.1 \pm 1.7$ & 58 & $11.9 \pm 1.5$ & 0.422 \\
\hline Platelet $\left(\times 10^{3} / \mu \mathrm{L}\right)$ & & 96 & $335.7 \pm 143.9$ & 58 & $353.0 \pm 113.3$ & 0.437 \\
\hline NLR & & 96 & $4.7 \pm 4.3$ & 58 & $5.1 \pm 3.1$ & 0.520 \\
\hline PLR & & 96 & $262.1 \pm 201.9$ & 58 & $336.4 \pm 236.7$ & 0.040 \\
\hline LMR & & 96 & $3.9 \pm 2.5$ & 58 & $3.2 \pm 1.8$ & 0.036 \\
\hline
\end{tabular}

$P$-values for comparison of mean difference for continuous variables were obtained by t-test;

$P$-values for independent test for categorical variables were obtained by Chi-squared test.

SD, standard deviation; FIGO, The International Federation of Gynecology and Obstetrics; CA125, cancer antigen 125; WBC, white blood cell; ANC, absolute neutrophil count; AMC, absolute monocyte count; AEC, absolute eosinophil count; ALC, absolute lymphocyte count; Hb, hemoglobin; NLR, neutrophil-lymphocyte ratio; PLR, platelet-lymphocyte ratio; LMR, lymphocyte-monocyte ratio.

Table 3. Relationship between patient characteristics and suboptimal cytoreduction in the 154 patients with epithelial ovarian cancer.

\begin{tabular}{|c|c|c|c|c|}
\hline \multirow[b]{2}{*}{ Variable } & \multicolumn{2}{|l|}{ Univariate } & \multicolumn{2}{|l|}{ Multivariate } \\
\hline & HR (95\% CI) & $P$-value & HR $(95 \% \mathrm{CI})$ & $P$-value \\
\hline Age (years) ( $\leq 73$ vs. $>73)$ & $4.75(1.58-14.29)$ & 0.0056 & $5.98(1.79-20.03)$ & 0.0037 \\
\hline Histology (serous vs. non-serous) & $0.87(0.40-1.87)$ & 0.7160 & & \\
\hline Histologic grade (G1 vs. G2/G3) & $1.07(0.34-3.36)$ & 0.9112 & & \\
\hline FIGO stage (III vs. IV) & $3.18(1.23-8.23)$ & 0.0172 & & \\
\hline Ascites (no vs. yes) & $1.56(0.68-3.55)$ & 0.2947 & & \\
\hline CA125 (unit/mL) ( $\leq 1467$ vs. >1467) & $2.48(1.19-5.14)$ & 0.0149 & $2.55(1.13-5.77)$ & 0.0249 \\
\hline Albumin $(\mathrm{g} / \mathrm{dL})(\leq 3.4$ vs. $>3.4)$ & $0.52(0.26-1.07)$ & 0.0769 & & \\
\hline WBC $($ per $\mu \mathrm{L})(\leq 8160$ vs. $>8160)$ & $0.45(0.22-0.92)$ & 0.0289 & $0.32(0.14-0.72)$ & 0.0062 \\
\hline ANC $($ per $\mu \mathrm{L})(\leq 3892.8$ vs. $>3892.8)$ & $1.98(0.88-4.45)$ & 0.0997 & & \\
\hline $\mathrm{AMC}($ per $\mu \mathrm{L})(\leq 512.1$ vs. 512.1$)$ & $0.58(0.29-1.18)$ & 0.1324 & & \\
\hline AEC (per $\mu \mathrm{L})(\leq 131.3$ vs. $>131.3)$ & $0.53(0.25-1.11)$ & 0.0920 & & \\
\hline ALC $($ per $\mu \mathrm{L})(\leq 1858.6$ vs. $>1858.6)$ & $0.30(0.11-0.77)$ & 0.0122 & & \\
\hline $\mathrm{Hb}(\mathrm{g} / \mathrm{dL})(\leq 11.2$ vs. $>11.2)$ & $0.53(0.26-1.10)$ & 0.0885 & & \\
\hline Platelet $\left(\times 10^{3} / \mu \mathrm{L}\right)(\leq 290$ vs. $>290)$ & $1.88(0.94-3.76)$ & 0.0758 & & \\
\hline NLR $(\leq 5.35$ vs. $>5.35)$ & $2.32(1.13-4.79)$ & 0.0225 & & \\
\hline PLR $(\leq 270.70$ vs. $>270.70)$ & $2.98(1.52-5.86)$ & 0.0015 & & \\
\hline $\operatorname{LMR}(\leq 3.75$ vs. $>3.75)$ & $0.27(0.13-0.58)$ & 0.0006 & $0.27(0.12-0.61)$ & 0.0015 \\
\hline
\end{tabular}

Hazard ratio was obtained by Cox's proportional hazard model.

The two groups in the parenthesis are categorized by the cutoff values derived from receiver operating characteristic curve analysis.

$\mathrm{HR}$, hazard ratio; CI, confidence interval; FIGO, The International Federation of Gynecology and Obstetrics; CA125, cancer antigen 125; WBC, white blood cell; ANC, absolute neutrophil count; AMC, absolute monocyte count; AEC, absolute eosinophil count; ALC, absolute lymphocyte count; Hb, hemoglobin; NLR,

neutrophil-lymphocyte ratio; PLR, platelet-lymphocyte ratio; LMR, lymphocyte-monocyte ratio. 


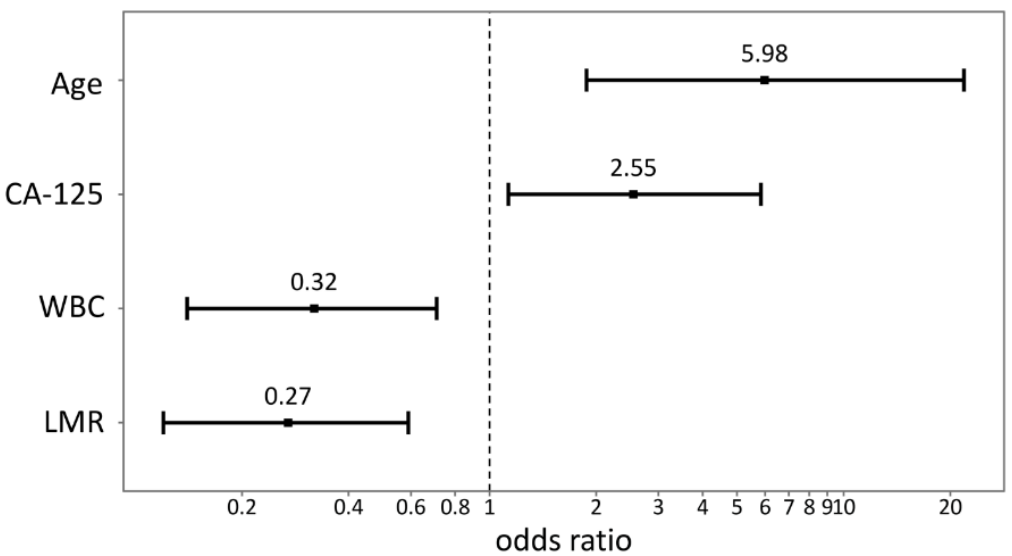

Figure 1. Plots of odds ratio. CA125, cancer antigen 125; WBC, white blood cell; LMR, lymphocyte-monocyte ratio.

Table 4. Results of cytoreduction according to age, WBC, and CA125 in the 154 patients with epithelial ovarian cancer.

\begin{tabular}{|c|c|c|c|c|c|c|}
\hline & \multicolumn{3}{|l|}{ LMR $\leq 3.75$} & \multicolumn{3}{|l|}{ LMR $>3.75$} \\
\hline & $\begin{array}{l}\text { Optimal cytoreduction, } \\
n(\%)\end{array}$ & $\begin{array}{l}\text { Suboptimal cytoreduction, } \\
n(\%)\end{array}$ & $P$-value & $\begin{array}{l}\text { Optimal cytoreduction, } \\
n(\%)\end{array}$ & $\begin{array}{l}\text { Suboptimal cytoreduction, } \\
n(\%)\end{array}$ & $P$-value \\
\hline Age (years) & & & 0.012 & & & 1.000 \\
\hline$\leq 73$ & $47(57.3)$ & $35(42.7)$ & & $44(80.0)$ & $11(20.0)$ & \\
\hline$>73$ & $2(15.4)$ & $11(84.6)$ & & $3(75.0)$ & $1(25.0)$ & \\
\hline WBC (per $\mu \mathrm{L})$ & & & 0.023 & & & 0.494 \\
\hline$\leq 8160$ & $24(41.4)$ & $34(58.6)$ & & $32(76.2)$ & $10(23.8)$ & \\
\hline$>8160$ & $25(67.6)$ & $12(32.4)$ & & $15(88.2)$ & $2(11.8)$ & \\
\hline CA125 (unit/mL) & & & 0.079 & & & 0.827 \\
\hline$\leq 1467$ & $38(58.5)$ & $27(41.5)$ & & $39(81.2)$ & $9(18.8)$ & \\
\hline$>1467$ & $11(36.7)$ & $19(63.3)$ & & $8(72.7)$ & $3(27.3)$ & \\
\hline
\end{tabular}

WBC, white blood cell; CA125, cancer antigen 125; LMR, lymphocyte-monocyte ratio.

Subgroup analyses were performed to evaluate the correlation between outcomes of cytoreductive surgery and age, CA125, or WBC in both low- and high-LMR level groups. There was significant correlation between cytoreductive outcome and age $(P=0.012)$ or WBC count $(P=0.023)$ in the low-LMR group. However, in the high-LMR group, there was no significant correlation between cytoreductive outcome and age or WBC. Finally, the correlation between cytoreductive outcome and CA125 level was not significant in either the low- or high-LMR group (Table 4).

\section{Discussion}

Ovarian cancer remains one of the leading causes worldwide of death from cancer in women [1]. One of the major reasons for the poor survival rate of ovarian cancer is that most ovarian cancers are identified at an advanced stage [2], and patients experience disease recurrence at some point in their lives [27]. Aggressive optimal cytoreductive surgery plus postoperative administration of chemotherapy is the currently recommended treatment for advanced stage ovarian cancer [3]. Of note, the achievement of optimal cytoreduction is one of the most important factors influencing prognosis.

The definition of optimal cytoreduction remains a subject of considerable controversy. It has varied from a maximum diameter of residual tumor of $2 \mathrm{~cm}$ to no residual mass whatsoever [28]. The GOG currently designates optimal surgical cytoreduction as a largest residual tumor mass of $1 \mathrm{~cm}$ or less in maximum diameter [25]. The impact of the results of cytoreductive surgery on survival has been demonstrated in a systematic review of eleven studies, which found that optimal cytoreduction with a largest residual tumor mass of $1 \mathrm{~cm}$ or less in maximum diameter correlates with an improvement in OS [29]. In this work, we defined optimal cytoreduction according to the definition of the GOG [25], and suboptimal cytoreduction as surgical exploration with residual tumor of more than $1 \mathrm{~cm}$ in maximum diameter.

Although several previous studies have reported various kinds of potential tools for predicting the results of cytoreductive surgery, controversial results have also been reported, and as such there is no scientific evidence to support those tools as predictors 
for the results of cytoreductive surgery. Consequently, there is at present no strong predictor with performance adequate for clinical practice.

LMR has been proposed by many researchers as a prognostic factor for the survival of patients with lymphomas [23] or various types of malignancies including ovarian cancers [24]. In our previous report [24], along with age and FIGO stage, LMR was identified to be the most significant prognosticator for OS. Although the mechanisms underlying the relationship between lower LMR and poor survival rates in many different types of tumors have not been fully clarified, some suggestions can be derived by considering the natures of lymphocytes and monocytes in peripheral blood. Lymphocytes have been suggested to play a crucial role in the fight against cancer, and the lymphocytopenia has long been identified as a poor prognostic factor for OS in various types of cancers. As well, an increase in monocyte counts in the peripheral blood has been recognized as a poor prognostic factor in different types of cancers $[30,31]$. In the present work, LMR was found to be the strongest predictor of suboptimal cytoreduction, with a cutoff value of 3.75 based on ROC curve analysis. In our previous study on ovarian cancer, the best LMR cutoff value for OS was found to be 2.07 based on ROC curve analysis. When performing logistic regression analysis with a cutoff value of 2.07, LMR was not found to be a predictor of suboptimal cytoreduction. In the present work, the value of LMR as a predictor for suboptimal surgical cytoreduction was assessed together with NLR and PLR. The impact of NLR on progression-free survival (PFS) [32, 33] and OS [33-35] has been reported in ovarian cancers. In addition, the preoperative PLR has been demonstrated to have prognostic impact on the survival of patients with ovarian cancers [36, 37]. Although all three potential inflammatory biomarkers were found to be significant predictors for suboptimal cytoreduction in univariate analysis, LMR was the only significant predictor in multivariate analysis.

In the present work, age (cutoff of 73 years) was demonstrated to be a predictor of suboptimal cytoreduction. Several other researchers have also stressed the importance of age, and have reported that cytoreduction was easier to accomplish in younger patients. The cutoff values of those studies were 70 [8] and 60 years [9]. However, the value of age as a predictor of cytoreduction is not conclusive. Several studies have reported opposite results showing that outcome of cytoreductive surgery is not dependent on age. The cutoff values of those studies were 70 [38], 62 [17], and 55 years [21].

In this study, preoperative CA125 (cutoff of 1467 units $/ \mathrm{mL}$ ) was significantly associated with risk of suboptimal cytoreduction. Several investigators have empirically demonstrated a correlation between preoperative CA125 level and the results of cytoreductive surgery. The most common cutoff value of those studies was 500 units/mL [9, 12, 13, 15], although a cutoff of 586 units/mL has also been reported [14]. However, the value of CA125 as a predictor of cytoreductive outcome is also not conclusive. Several studies have reported opposite results indicating that CA125 is not predictive of the results of cytoreductive surgery. The cutoff values of those studies were diverse, including 1791 [17], 2145 [17], 756 [21], and 500 units/mL [39, 40]. When we applied 500 units/mL as a cutoff value, CA125 was not predictive of cytoreductive outcome.

In the present study, an association between preoperative WBC (cutoff of $8160 / \mu \mathrm{L}$ ) count and suboptimal cytoreduction was found. The results of the current work do not agree well with a previous report of So et al., which found that optimal surgical cytoreduction was attained in $67.4 \%$ and $65.5 \%$ of patients with EOC in groups with $\mathrm{WBC} \leq 10000 / \mu \mathrm{L}$ and $\mathrm{WBC}>10000 / \mu \mathrm{L}$, respectively $(P=0.857)$ [41]. When we applied $10000 / \mu \mathrm{L}$ as a cutoff value, WBC was not a predictor for the outcomes of cytoreductive surgery.

In this work, we also evaluated the value of histologic grade before cytoreduction as a predictor for cytoreductive outcome. In a study by Heintz et al., it was much easier to accomplish optimal surgical cytoreduction in patients with low-grade tumors [8]. However, in the present work, histologic grade was not predictive of the results of cytoreductive surgery.

In the current study, we did not evaluate the value of specific laparoscopic features as a reliable tool for predicting the outcomes of cytoreductive surgery. It is impossible to determine whether a patient can be optimally debulked until the cytoreduction procedure is underway, thus limiting the value of laparoscopic features as a preoperative predictor for suboptimal cytoreduction [16]. We also did not include specific findings of radiologic studies, including CT scan $[9,17,18]$ and PET/CT [19-21], as predictors for cytoreductive outcomes. The reason is that use of specific imaging results before surgical resection is difficult in clinical application, and they have not been validated in large prospective randomized trials [42].

The strength of the present study is that it represents the first attempt to evaluate the role of preoperative LMR, a simple and low-cost peripheral blood examination, as a predictor for risk of suboptimal cytoreduction for EOC. In addition, we evaluated the value of LMR together with well-known systemic inflammation-based biomarkers 
such as NLR and PLR. Finally, the current study was a multiple-institution research study. However, the limitations of the study included its characteristics of design, being a retrospective study, and the relatively small number of patients that met the inclusion criteria of the study. In addition, as LMR is a non-specific marker of inflammatory activity, LMR values may have been affected by the other inflammatory conditions, a variety of systemic diseases, or baseline characteristics of enrolled patients [43]. Finally, we could not evaluate the role of LMR compared with other inflammation-based markers such as erythrocyte sedimentation rate and C-reactive protein due to the considerable number of missing values.

In conclusion, this study evaluated, for the first time, the value of LMR as a predictor for the outcome of primary surgical cytoreduction in EOC patients, and found that LMR is a clinically reliable predictor for suboptimal cytoreduction, along with age, CA125 level, and WBC count. The results of our study reveal a possible role of LMR, as an inflammatory biomarker, that may provide additional prognostic information beyond the biological parameters of the tumor. Additional large-scale investigations should be performed to improve understanding of the role.

\section{Acknowledgements}

This study was supported by a grant from the National R\&D Program for Cancer Control, Ministry for Health, Welfare and Family affairs, Republic of Korea (0920050).

\section{Competing Interests}

The authors have declared that no competing interest exists.

\section{References}

1. Siegel R, Naishadham D, Jemal A. Cancer statistics, 2013. CA. 2013;63:11-30.

2. Vergote I, Trope CG, Amant F, Kristensen GB, Ehlen T, Johnson N, et al. Neoadjuvant chemotherapy or primary surgery in stage IIIC or IV ovarian cancer. The New England journal of medicine. 2010;363:943-53.

3. Cannistra SA. Cancer of the ovary. The New England journal of medicine. 2004;351:2519-29.

4. Hoskins WI, McGuire WP, Brady MF, Homesley HD, Creasman WT, Berman $\mathrm{M}$, et al. The effect of diameter of largest residual disease on survival after primary cytoreductive surgery in patients with suboptimal residual epithelial ovarian carcinoma. American journal of obstetrics and gynecology. 1994:170:974-9.

5. Hou JY, Kelly MG, Yu H, McAlpine JN, Azodi M, Rutherford TJ, et al. Neoadjuvant chemotherapy lessens surgical morbidity in advanced ovarian cancer and leads to improved survival in stage IV disease. Gynecol Oncol. 2007;105:211-7

6. van der Burg ME, Vergote I. The role of interval debulking surgery in ovarian cancer. Current oncology reports. 2003;5:473-81.

7. Stashwick C, Post MD, Arruda JS, Spillman MA, Behbakht K, Davidson SA, et al. Surgical risk score predicts suboptimal debulking or a major perioperative complication in patients with advanced epithelial ovarian, fallopian tube, or primary peritoneal cancer. Int J Gynecol Cancer. 2011:21:1422-7.

8. Heintz AP, Hacker NF, Berek JS, Rose TP, Munoz AK, Lagasse LD. Cytoreductive surgery in ovarian carcinoma: feasibility and morbidity. Obstet Gynecol. 1986;67:783-8.

9. Suidan RS, Ramirez PT, Sarasohn DM, Teitcher JB, Mironov S, Iyer RB, et al. A multicenter prospective trial evaluating the ability of preoperative computed tomography scan and serum CA-125 to predict suboptimal cytoreduction at primary debulking surgery for advanced ovarian, fallopian tube, and peritoneal cancer. Gynecol Oncol. 2014;134:455-61.

10. Berchuck A, Iversen ES, Lancaster JM, Dressman HK, West M, Nevins JR, et al. Prediction of optimal versus suboptimal cytoreduction of advanced-stage serous ovarian cancer with the use of microarrays. American journal of obstetrics and gynecology. 2004;190:910-25.

11. Ferrandina G, Fagotti A, Salerno MG, Natali PG, Mottolese M, Maneschi F, et al. p53 overexpression is associated with cytoreduction and response to chemotherapy in ovarian cancer. British journal of cancer. 1999;81:733-40.

12. Chi DS, Venkatraman ES, Masson V, Hoskins WJ. The ability of preoperative serum CA-125 to predict optimal primary tumor cytoreduction in stage III epithelial ovarian carcinoma. Gynecol Oncol. 2000;77:227-31.

13. Vorgias G, Iavazzo C, Savvopoulos P, Myriokefalitaki E, Katsoulis M, Kalinoglou N, et al. Can the preoperative Ca-125 level predict optimal cytoreduction in patients with advanced ovarian carcinoma? A single institution cohort study. Gynecol Oncol. 2009;112:11-5.

14. Brockbank EC, Ind TE, Barton DP, Shepherd JH, Gore ME, A'Hern R, et al. Preoperative predictors of suboptimal primary surgical cytoreduction in women with clinical evidence of advanced primary epithelial ovarian cancer. Int J Gynecol Cancer. 2004;14:42-50.

15. Kang S, Kim TJ, Nam BH, Seo SS, Kim BG, Bae DS, et al. Preoperative serum CA-125 levels and risk of suboptimal cytoreduction in ovarian cancer: a meta-analysis. J Surg Oncol. 2010;101:13-7.

16. Fagotti A, Ferrandina G, Fanfani F, Garganese G, Vizzielli G, Carone V, et al. Prospective validation of a laparoscopic predictive model for optimal cytoreduction in advanced ovarian carcinoma. American journal of obstetrics and gynecology. 2008;199:642.e1-6.

17. MacKintosh ML, Rahim R, Rajashanker B, Swindell R, Kirmani BH, Hunt J, et al. CT scan does not predict optimal debulking in stage III-IV epithelial ovarian cancer: a multicentre validation study. Journal of obstetrics and gynaecology. 2014;34:424-8

18. Borley J, Wilhelm-Benartzi C, Yazbek J, Williamson R, Bharwani N, Stewart V, et al. Radiological predictors of cytoreductive outcomes in patients with advanced ovarian cancer. BJOG. 2015:122·843-9.

19. Alessi A, Martinelli F, Padovano B, Serafini G, Lorusso D, Lorenzoni A, et al. FDG-PET/CT to predict optimal primary cytoreductive surgery in patients with advanced ovarian cancer: preliminary results. Tumori. 2016;102:103-7.

20. Vargas HA, Burger IA, Goldman DA, Micco M, Sosa RE, Weber W, et al. Volume-based quantitative FDG PET/CT metrics and their association with optimal debulking and progression-free survival in patients with recurrent ovarian cancer undergoing secondary cytoreductive surgery. European radiology. 2015;25:3348-53

21. Shim SH, Kim DY, Seo MJ, Lee SW, Park JY, Lee JJ, et al. Preoperative fluorine 18 fluorodeoxyglucose tumoral uptake ratio between upper and lower abdomen in primary advanced-stage ovarian cancer. Int J Gynecol Cancer. 2013;23:1383-92.

22. Farias-Eisner R, Teng F, Oliveira M, Leuchter R, Karlan B, Lagasse LD, et al. The influence of tumor grade, distribution, and extent of carcinomatosis in minimal residual stage III epithelial ovarian cancer after optimal primary cytoreductive surgery. Gynecol Oncol. 1994;55:108-10.

23. Porrata LF, Ristow K, Colgan JP, Habermann TM, Witzig TE, Inwards DJ, et al Peripheral blood lymphocyte/monocyte ratio at diagnosis and survival in classical Hodgkin's lymphoma. Haematologica. 2012;97:262-9.

24. Eo WK, Chang $\mathrm{HI}$, Kwon $\mathrm{SH}, \mathrm{Koh} \mathrm{SB}$, Kim $\mathrm{YO}$, Ji $\mathrm{YI}$, et al. The Lymphocyte-Monocyte Ratio Predicts Patient Survival and Aggressiveness of Ovarian Cancer. Journal of Cancer. 2016;7:289-96.

25. Fader AN, Rose PG. Role of surgery in ovarian carcinoma. Journal of clinical oncology. 2007;25:2873-83.

26. Hacker NF, Berek JS, Lagasse LD, Nieberg RK, Elashoff RM. Primary cytoreductive surgery for epithelial ovarian cancer. Obstet Gynecol. 1983;61:413-20.

27. Bandera EV, Kushi LH, Rodriguez-Rodriguez L. Nutritional factors in ovarian cancer survival. Nutr Cancer. 2009;61:580-6.

28. Vergote I, van Gorp T, Amant F, Leunen K, Neven P, Berteloot P. Timing of debulking surgery in advanced ovarian cancer. Int J Gynecol Cancer. 2008;18 Suppl 1:11-9.

29. Elattar A, Bryant A, Winter-Roach BA, Hatem M, Naik R. Optimal primary surgical treatment for advanced epithelial ovarian cancer. Cochrane Database Syst Rev. 2011:Cd007565

30. Huang JJ, Li YJ, Xia Y, Wang Y, Wei WX, Zhu YJ, et al. Prognostic significance of peripheral monocyte count in patients with extranodal natural killer/T-cell lymphoma. BMC cancer. 2013;13:222

31. Qi Q, Geng Y, Sun M, Wang P, Chen Z. Clinical implications of systemic inflammatory response markers as independent prognostic factors for advanced pancreatic cancer. Pancreatology 2015:15:145-50.

32. Kim HS, Choi HY, Lee M, Suh DH, Kim K, No JH, et al. Systemic Inflammatory Response Markers and CA-125 Levels in Ovarian Clear Cell Carcinoma: A Two Center Cohort Study. Cancer Res Treat. 2016:48:250-8.

33. Wang Y, Liu P, Xu Y, Zhang W, Tong L, Guo Z, et al. Preoperative neutrophil-to-lymphocyte ratio predicts response to first-line platinum-based chemotherapy and prognosis in serous ovarian cancer. Cancer Chemother Pharmacol. 2015;75:255-62.

34. Cho H, Hur HW, Kim SW, Kim SH, Kim JH, Kim YT, et al. Pre-treatment neutrophil to lymphocyte ratio is elevated in epithelial ovarian cancer and 
predicts survival after treatment. Cancer immunology, immunotherapy. 2009;58:15-23.

35. Williams KA, Labidi-Galy SI, Terry KL, Vitonis AF, Welch WR, Goodman A, et al. Prognostic significance and predictors of the neutrophil-to-lymphocyte ratio in ovarian cancer. Gynecol Oncol. 2014;132:542-50.

36. Asher V, Lee J, Innamaa A, Bali A. Preoperative platelet lymphocyte ratio as an independent prognostic marker in ovarian cancer. Clinical \& translational oncology. 2011;13:499-503.

37. Supoken A, Kleebkaow P, Chumworathayi B, Luanratanakorn S, Kietpeerakool C. Elevated preoperative platelet to lymphocyte ratio associated with decreased survival of women with ovarian clear cell carcinoma. Asian Pacific journal of cancer prevention. 2014;15:10831-6.

38. Wright JD, Herzog TJ, Powell MA. Morbidity of cytoreductive surgery in the elderly. American journal of obstetrics and gynecology. 2004;190:1398-400.

39. Barlow TS, Przybylski M, Schilder JM, Moore DH, Look KY. The utility of presurgical CA125 to predict optimal tumor cytoreduction of epithelial ovarian cancer. Int J Gynecol Cancer. 2006;16:496-500.

40. Chi DS, Zivanovic O, Palayekar MJ, Eisenhauer EL, Abu-Rustum NR, Sonoda $\mathrm{Y}$, et al. A contemporary analysis of the ability of preoperative serum CA-125 to predict primary cytoreductive outcome in patients with advanced ovarian, tubal and peritoneal carcinoma. Gynecol Oncol. 2009;112:6-10.

41. So KA, Hong JH, Jin HM, Kim JW, Song JY, Lee JK, et al. The prognostic significance of preoperative leukocytosis in epithelial ovarian carcinoma: a retrospective cohort study. Gynecol Oncol. 2014;132:551-5.

42. Axtell AE, Lee MH, Bristow RE, Dowdy SC, Cliby WA, Raman S, et al. Multi-institutional reciprocal validation study of computed tomography predictors of suboptimal primary cytoreduction in patients with advanced ovarian cancer. Journal of clinical oncology. 2007;25:384-9.

43. Madjid M, Fatemi O. Components of the complete blood count as risk predictors for coronary heart disease: in-depth review and update. Texas Heart Institute journal. 2013;40:17-29. 